\title{
FGFR4 Gly388Arg Polymorphism Affects the Progression of Gastric Cancer by Activating STAT3 Pathway to Induce Epithelial to Mesenchymal Transition
}

\author{
Yanwei Ye, $\mathrm{PhD}^{1}$ \\ Jie Li, MD² \\ Dongbao Jiang, $M D^{3}$ \\ Jingjing Li, $\mathrm{PhD}^{4}$ \\ Chuangfeng Xiao, MD \\ Yingze Li, MD ${ }^{1}$ \\ Chao Han, $\mathrm{PhD}^{5}$ \\ Chunlin Zhao, $\mathrm{PhD}^{1}$
}

${ }^{1}$ Department of Gastrointestinal Surgery and Institute of Clinical Medicine, The First Affiliated Hospital of Zhengzhou University, Zhengzhou, ${ }^{2}$ Department of General Surgery, Tongchuan People's Hospital, Shanxi, ${ }^{3}$ Department of Thyroid, and Breast Surgery, Xinxiang Central Hospital, Xinxiang, Departments of ${ }^{4}$ Infectious Disease and ${ }^{5}$ Pharmacy, The First Affiliated Hospital of Zhengzhou University, Zhengzhou, China

Correspondence: Yanwei Ye, PhD

Department of Gastrointestinal Surgery and Institute of Clinical Medicine, The First Affiliated Hospital of Zhengzhou University, 1 Eastern Jian-She Road, Zhengzhou,

Henan 450052, China

Tel: 86-13838389763

Fax: 86-037167967236

E-mail: yeyanwei66@163.com

Received February 24, 2020

Accepted May 24, 2020

Published Online May 25, 2020

*Yanwei Ye and Jie Li contributed equally to this work.

\begin{abstract}
Purpose
Fibroblast growth factor receptor 4 (FGFR4) plays a critical role in cancer progression involving in tumor proliferation, invasion, and metastasis. This study clarified the role of FGFR4Arg388 variant in gastric cancer (GC), and more importantly highlighted the possibility of this single nucleotide polymorphism (SNP) as potential therapeutic targets.
\end{abstract}

\section{Materials and Methods}

FGFR4 polymorphism was characterized in advanced GC patients to perform statistical analysis. FGFR4-dependent signal pathways involving cell proliferation, invasion, migration, and resistance to oxaliplatin (OXA) in accordance with the SNP were also assessed in transfected GC cell lines.

\section{Results}

Among 102 GC patients, the FGFR4-Arg388 patients showed significantly higher tumor stage $(p=0.047)$ and worse overall survival $(p=0.033)$ than the Gly388 patients. Immunohistochemical results showed that FGFR4-Arg388 patients were more likely to have higher vimentin $(p=0.025)$ and $p-$ STAT3 $(p=0.009)$ expression compared with FGFR4-Gly388 patients. In transfected GC cells, the overexpression of FGFR4-Arg388 variant increased proliferation and invasion of GC cells, increasing resistance of GC cells to OXA compared with cells overexpressing the Gly388 allele.

\section{Conclusion}

The exploration mechanism may be through FGFR4-Arg388/STAT3/epithelial to mesenchymal transition axis regulating pivotal oncogenic properties of GC cells. The FGFR4-Arg388 variant may be a biomarker and a candidate target for adjuvant treatment of GC.

\section{Key words}

Fibroblast growth factor receptor 4, Gly388Arg polymorphism, Gastric neoplasms, STAT3, Epithelial to mesenchymal transition

\section{Introduction}

The U.S. Food and Drug Administration (FDA) approved herceptin and ramucirumab for the treatment of advanced gastric cancer (GC), while the overall effect was not satisfactory. Therefore, whether there are new molecular markers to be pivotal in the occurrence and development of GC, as well as further targeted drugs worthy of research and development, will become a further breakthrough in the field of GC research.
In recent years, a mass of molecular changes having an important role in the pathogenesis and prognosis of tumor patients have been confirmed. The fibroblast growth factor receptor 4 (FGFR4) has been proven therapeutic potential in a variety of cancer types [1], which has been connected with process and prognosis in several kinds of cancer, involving GC [2-4]. FGFR-Arg388 variant (rs351855 at the genotype level), contains an amino acid rep-lacement of an arginine for a glycine at codon 388. FGFR4-Arg388 variant plays a role in susceptibility to oral squamous cell carcinoma, 
pituitary tumors, and neuroblastoma [5-7]. FGFR4-Arg388 variant was a new independent prognostic factor and correlated with tumor poorer prognosis in various tumors [8-10]. FGFR4-Arg388 variant also affects the tumor biological behavior. For example, FGFR4-Gly388 tumors express incremental prolactin and less growth hormone, whereas tumors holding the polymorphic variant of FGFR4-Arg388 express enhancive growth hormone with respect to prolactin [11].

The FGFR4-Arg388 variant has been declared to be associated with multiplesignaling pathways. Ulaganathan and Ullrich [12] believe FGFR4-Arg388 variant might expose a proximal STAT3 binding site and have confirmed that FGFR4-Arg388 variant could induce enhanced STAT3 signal. The FGFR4Arg388 allele produces a receptor variant that preferentially promotes STAT3 / 5 signaling, transcriptionally inducing Grb14 in pancreatic endocrine cells to promote insulin secretion [13]. Moreover, FGFR4-Arg388 variant has been related to epithelial to mesenchymal transition (EMT), inducing expression of EMT-associated proteins [14,15]. The FGFR4 variant induces STAT3 activation and the expression of EMT-related genes, thereby causing pro-oncogenic effects in lung cancer in vitro and in vivo [16]. In the current research, we aimed to explore the potential effect of the FGFR4-Arg388 variant on GC biological behavior, its resistance to oxaliplatin (OXA), its impact on patients' pathological features and prognosis in this setting, whether it was caused by EMT and STAT3 pathways, with exploration of the relationship creatively.

\section{Materials and Methods}

\section{Patients}

Collection of 102 consecutive primary GC tissue samples was in the First Affiliated Hospital of Zhengzhou University (Zhengzhou, China). All patients were diagnosed and treated at this hospital. All GC patients without any chemotherapy, radiotherapy, and other therapies prior to radical gastrectomy. All cases were pathologically recorded and approved by the hospital ethics committee to record personal files for clinical data. Staging must be based on the American Joint Committee on Cancer (AJCC) tumor, lymph node metastasis system (TNM) staging for GC staging (7th edition, 2010) [17]. Follow-up data were acquired by telephone, letter acquisition, and outpatient clinical database. The survival time was calculated based on the date from the completion of the surgery to the date of death or the date of the last follow-up. Follow-up time was from January 2010 to December 2017.

\section{Immunohistochemical staining}

The expression of FGFR4, E-cadherin (E-cad), vimentin (Vim), STAT3, and p-STAT3 in paraffin-embedded tumor specimens of all selected patients was detected by immuno- histochemistry. The concentrations of antibodies and positive sites were as follows: anti-FGFR4, dilution 1:500, positive site was cytoplasm; anti-E-cad, dilution 1:200, positive site was cell membrane; anti-Vim, dilution 1:500, positive site was cytoplasm; anti-STAT3, dilution of 1:100, the positive sites were the nucleus and cytoplasm; anti-p-STAT3, dilution of 1:200, the positive sites were the nucleus and cytoplasm; the negative control (NC) was achieved by incubating the parallel slides to omit the accordingly primary antibody.

\section{Immunohistochemical staining scoring}

Slides were semi-quantitatively evaluated by two independent pathologists who were blinded to the patient's clinical data when scoring the immunohistochemical results of the archival tissue samples. Cytoplasmic FGFR4 immunostaining was scored (quaternary system): negative (-), low $(+)$, intermediate $(++)$, and high expression $(+++)$ [18]. Finally, the percentage of positive staining cells was scored according to the intensity score of each part: Vim of positive tumor cells dispersed throughout the section, constituting at least $10 \%$ [19]; When $>10 \%$ of tumor membranes were stained, E-cad expression was positive [20]. Overexpression of STAT3 and p-STAT3 were defined as nuclear staining of more than $30 \%$ of tumor cells in GC [21].

\section{Genotyping}

Genomic DNA was removed from the 102 tumor tissue stored in $4 \%$ paraformaldehyde. A 120-bp fragment possessing the Gly388Arg polymorphism was polymerase chain reaction (PCR) amplified using polymerized forward primer, 5'-CAGTACCTGTCGGGCCAGAG-3'; reverse, 5'-CTTGGCTGTGCTCCTGCTG-3'. One microliter of PCR products were labeled with the BigDye sequencing kit (Applied Biosystems, Foster City, CA) and the recommended operating conditions were on the basis of the manufacturer's instructions. The sequence data were interpreted with the DNSstar software (Fig. 1A).

\section{Cell lines and cell culture}

Human GC cell lines AGS, NCI-N87, MGC803, and MKN45 were obtained from the American Type Culture Collection. The BGC803 and SGC7901 cell lines were purchased from the Chinese Academy of Sciences, Science Bank of the typical Culture Collection (CBTCCCAS, Shanghai, China). Cell lines were cultured in RPMI-1640 medium (Gibco, Grand Island, NY) supplemented with $10 \%$ fetal bovine serum (FBS; Gibco), $100 \mathrm{U} / \mathrm{mL}$ penicillin and $100 \mu \mathrm{g} / \mathrm{mL}$ streptomycin (Caisson Labs, North Logan, UT) with a humidified atmosphere containing $5 \% \mathrm{CO}_{2}$ at $37^{\circ} \mathrm{C}$.

\section{Antibodies and reagents}

Rabbit monoclonal anti-E-cad (\#3195), anti-Vim (\#5741), 
A

GG

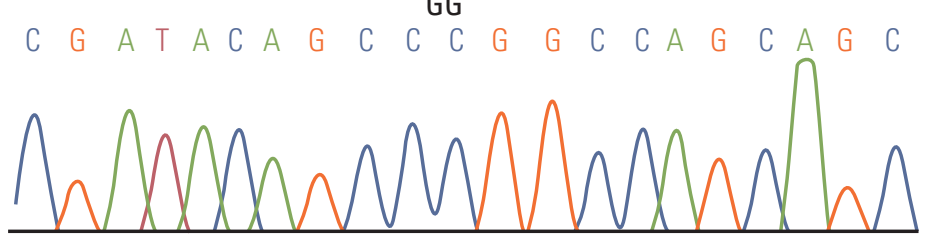

GA

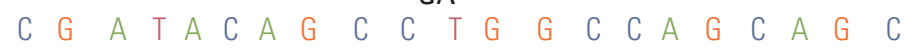

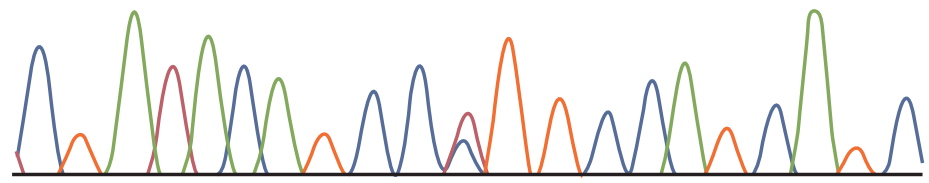

$\mathrm{AA}$

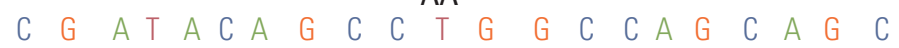

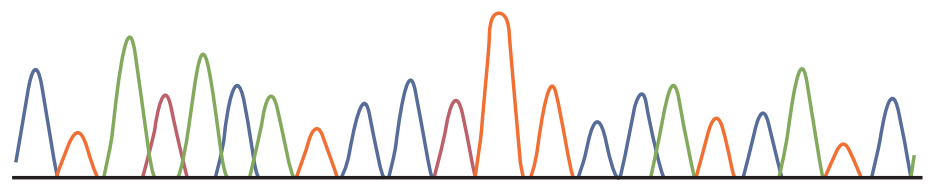

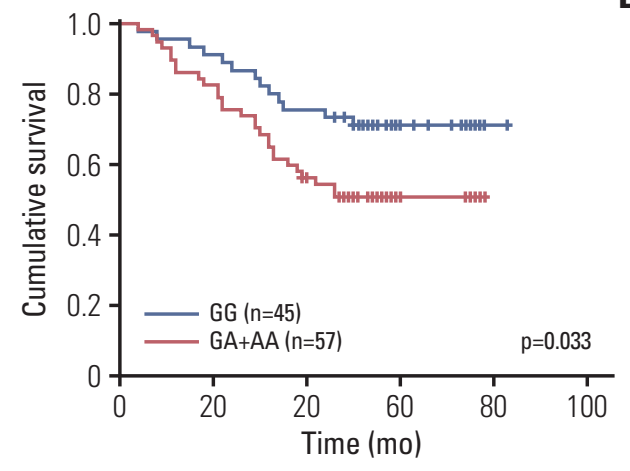
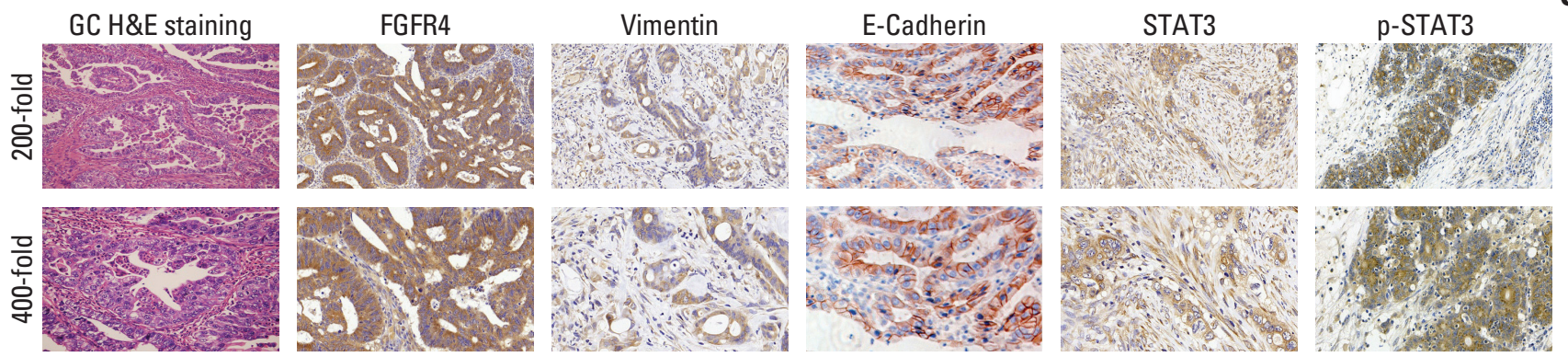

Fig. 1. (A) Gene sequencing including three specific fibroblast growth factor receptor 4 (FGFR4) Gly388Arg polymorphic genotype (including Gly / Gly, Gly/Arg, and Arg/Arg). (B) Significant difference was observed between patients with FGFR4-Gly388 allele and FGFR4-Arg388 variant among 102 gastric cancer (GC) patients after stratified Kaplan-Meier survival analysis. (C) Strong positive expressions of immunohistochemical markers in GC tissue were demonstrated. All H\&E staining and immunohistochemical pictures were amplified 200-fold (upper) and 400-fold (lower).

anti-p-STAT3 (\#9145), anti-caspase-3 (\#14220), and anticleaved caspase-3 (\#9664) as well as mouse monoclonal anti- $\beta$-actin (\#3700) and anti-STAT3 (\#9139) were all bought in Cell Signaling Technology (Beverly, MA). The anti-FGFR4 antibody was purchased by Proteintech (\#11098-1-AP) (Wuhan, China). Secondary horseradish peroxidase-conjugated antibodies were goat anti-mouse and goat anti-rabbit from Sigma-Aldrich Corp. (St. Louis, MO). AG490 was bought from Sigma-Aldrich Corporation (Shanghai, China). In addition, OXA came from our clinical trial group at the research center.

\section{Lentiviral overexpression vector construction and trans- fection}

Lentiviral overexpression vector construction and transfection (pHBLV-CMV-MCS-3flag-EF1-puro, including FGFR4-
Gly388, FGFR4-Arg388, and NC) were completed by Hanbio (Shanghai, China). The primer sequences of FGFR4-Gly388 were as follows: 5' -ggatctattccggtGaattcGCCACCATGCGGCTGCTGCTGGCCCTGTT-3' and 5'-CTTAAGCTTGGTACCGAggatccTGTCTGCACCCCAGACCCGAA-3'. The primer sequences of FGFR4-Arg388 were as follows: $5^{\prime}$-ggatctatttccggtGaattcGCCACCATGCGGCTGCTGCTGGCCCTGTT-3' and 5'-CTTAAGCTTGGTACCGAggatccTGTCTGCACCCCAGACCCGAA-3'. GC cells were seeded in a 24well plate at a density of $5 \times 10^{5}$ cells per well for 24 hours, then infected by adding the prepared virus according to the manufacturer's instructions. After the virus control group completely died under the action of puromycin, the cells of the experimental group were considered to be completely screened. 


\section{Reverse transcription PCR and quantitative real-time PCR}

Total RNA was collected from GC cell lines using TRIzol reagent. DNA synthesis kit (MBI, Fermentas, Canada) was reversely transcribed into cDNA of each RNA sample. Primers were: FGFR4, 5' -agatactcaaagacaacgcct-3' and 5' -cgcactccacgatcacgta- $3^{\prime} ; \beta$-actin, 5' -cacgatggaggggccggactcatc- $3^{\prime}$ and 5'-taaagacctctatgccaacacacagt-3'. Two Taq PCR MasterMix (Takara, Tokyo, Japan) was used for PCR amplification. FGFR4 annealing temperature for $57^{\circ} \mathrm{C}$. PCR products were electrophoresis with $2 \%$ agarose gel and stained with ethyl bromide. According to the instructions of Takara, the mRNA expression of FGFR4 in six GC cell lines was detected by quantitative real-time PCR (q-PCR). Repeat the experiment three times. Relative differences were calculated according to the comparative $\mathrm{Ct}$ method.

\section{Protein extraction and western blot}

Whole cell lysates were prepared using protein extraction reagents (Merck, Darmstadt, Germany). Protein samples boiled for 10 minutes (30 $\mu$ g per protein) were added to a $10 \%$ sodium dodecyl sulfate polyacrylamide gel for electrophoretic separation and transferred to a polyvinylidene difluoride (PVDF) membrane. The PVDF membrane was blocked in phosphate buffered saline (PBS) containing $0.05 \%$ Tween-20 and 5\% skim milk powder for 1 hour at room temperature, and incubated with primary antibody overnight at $4^{\circ} \mathrm{C}$. Then wash the PVDF membrane three times with PBS containing $0.05 \%$ Tween-20 and $1 \%$ skim milk powder. The PVDF membrane was then incubated with the secondary antibody for 1 hour at room temperature. The protein imprint was detected by an ECL detection system (ImageQuant LAS 3000, General Electric Co., Fairfield, CT). At least all samples were performed on three independent western blot analyses.

\section{Apoptosis assay}

GC cells, including mock, NC, and FGFR4-Gly388 and FGFR4-Arg388 overexpression groups were dealt with OXA at proper concentration (average value of $\mathrm{IC}_{50}$ of mock group and NC group) for 24 hours. Annexin V and propidium iodide (PI) were used for cell apoptosis detection by flow cytometry and fluorescence microscope (Nikon, Tokyo, Japan). Then cells were combined in $500 \mu \mathrm{L}$ annexin $\mathrm{V}$ binding buffer and incubated with $5 \mu \mathrm{L}$ Annexin V-PI in the dark and at room temperature for 15 minutes. After that, all samples were analyzed by FACS Calibur flow cytometry with CellQuest software (Thermo Fisher Scientific, Waltham, MA) and photographed under a fluorescence microscope.

\section{Cell proliferation assays}

Cell viability was assessed using Cell Counting Kit-8 (CCK-8) reagent (Dojindo Laboratories, Kumamoto, Japan) and EdU reagent (Ribobio, Guangzhou, China).

\section{1) CCK-8 proliferation assay}

One hundred microliters of cell medium containing 2,000 cells was seeded into each well of 96-well plates. After 1, 2, 3,4 , and 5 days of culture respectively, the supernatant was removed. The absorbance at $450 \mathrm{~nm}$ was measured using a microplate reader.

\section{2) Gradient concentration CCK-8 assays}

After culturing for 24 hours, cells were dealt with OXA at different concentrations ladder $(0,1,2,4,8,16,32,64,128$, and $256 \mu \mathrm{g})$. After 72 hours of culture, the supernatant was removed. The absorbance was measured at $450 \mathrm{~nm}$ with a microplate reader. Cell viability and $\mathrm{IC}_{50}$ were calculated for subsequent experiments.

\section{3) EdU proliferation assay}

Cells were seeded in 24 -well plates at $4 \times 10^{5}$ cells / well in $1 \mathrm{~mL}$ of culture medium. Each hole was incubated at room temperature for 30 minutes with $300-400 \mu \mathrm{L}$ cell stationary fluids (PBS containing 4\% paraformaldehyde). Two hundred microliters of $1 \times$ Apollo staining reaction solution was added to each well to mark EdU. Each well was incubated with $200 \mu \mathrm{L} 1 \times$ Hoechst 33342 reaction solutions at room temperature and decolorize shaker for 30 minutes for DNA staining. Under ultraviolet light, the cell nuclei were all stained blue, and the proliferating nuclei were stained red under a green light.

\section{Matrigel invasion and invasion assay}

The polycarbonate filter (8- $\mu$ m pore size) was covered with Matrigel gel (BD Biosciences, Franklin Lakes, NJ) at a concentration of $1 \mu \mathrm{g} / \mathrm{mL}$ and placed in a 24-well cell culture plate. Each well was inoculated $1 \times 10^{5}$ cells of $100 \mu \mathrm{L}$ of FBS-free medium in the upper part of the chamber, while the lower part of the chamber was filled with medium containing $20 \%$ FBS as a control. After incubation at $37^{\circ} \mathrm{C}$ for 72 hours, cells invaded with Matrigel membrane were fixed with $4 \%$ formaldehyde and stained with hematoxylin and eosin (H\&E) reagent. Wipe off the non-migrating cells on the upper side of the filter. Calculate at least 10 locations per filter. For cell scratch assays, SGC7901 and BGC803 cells (mock, NC,FGFR4-Gly388, and FGFR4-Arg388) in 6-well plates were scored with welldefined scratches and cultured for 24 hours. Obtain images under the fluorescence microscope at the same interval for 8 hours. The migration distance is quantified by Image-Pro Plus software (Media Cybemetics, Rockville, MD).

\section{Statistical analysis}

The connection between FGFR4 expression and clinicopathological factors as well as other immunohistochemical markers was assessed by chi-square test. Survival curves 
Table 1. Association analysis of the FGFR4 Gly388Arg polymorphism and clinicopathological parameters in gastric cancer patients

\begin{tabular}{|c|c|c|c|c|c|}
\hline Variable & $\begin{array}{c}\text { Total } \\
(n=102)\end{array}$ & $\begin{array}{c}\text { GG } \\
(n=45)\end{array}$ & $\begin{array}{c}\mathrm{GA}+\mathrm{AA} \\
(\mathrm{n}=57)\end{array}$ & $\begin{array}{c}\text { Pearson's } \\
\chi^{2} \text { value }\end{array}$ & p-value \\
\hline \multicolumn{6}{|l|}{ Age (yr) } \\
\hline$<60$ & 52 & 23 & 29 & 0.001 & $>0.99$ \\
\hline$\geq 60$ & 50 & 22 & 28 & & \\
\hline \multicolumn{6}{|l|}{ Sex } \\
\hline Male & 74 & 35 & 39 & 1.105 & 0.373 \\
\hline Female & 28 & 10 & 18 & & \\
\hline \multicolumn{6}{|l|}{ Tumor size (cm) } \\
\hline$<4$ & 67 & 30 & 37 & 0.004 & $>0.99$ \\
\hline$\geq 4$ & 34 & 15 & 19 & & \\
\hline \multicolumn{6}{|l|}{ Differentiation } \\
\hline $\mathrm{G} 1+\mathrm{G} 2$ & 44 & 16 & 28 & 1.887 & 0.227 \\
\hline G3+G4 & 58 & 29 & 29 & & \\
\hline \multicolumn{6}{|l|}{ T category } \\
\hline $\mathrm{T} 1+\mathrm{T} 2$ & 43 & 24 & 19 & 4.125 & 0.047 \\
\hline $\mathrm{T} 3+\mathrm{T} 4$ & 59 & 21 & 38 & & \\
\hline \multicolumn{6}{|l|}{$\mathrm{N}$ category } \\
\hline N0 & 47 & 29 & 28 & 0.607 & 0.436 \\
\hline $\mathrm{N} 1+\mathrm{N} 2+\mathrm{N} 3$ & 54 & 26 & 28 & & \\
\hline \multicolumn{6}{|l|}{ M category } \\
\hline M0 & 95 & 41 & 51 & 2.713 & 0.130 \\
\hline M1 & 7 & 1 & 6 & & \\
\hline \multicolumn{6}{|l|}{ Borrmann type } \\
\hline Local type (early GC+I+II) & 50 & 15 & 35 & 3.494 & 0.071 \\
\hline Infiltrating type (III+IV) & 52 & 25 & 27 & & \\
\hline \multicolumn{6}{|l|}{ Lauren type } \\
\hline Intestinal type & 45 & 17 & 28 & 0.070 & 0.840 \\
\hline Diffuse infiltration type & 57 & 23 & 34 & & \\
\hline \multicolumn{6}{|l|}{ WHO type } \\
\hline Tubular adenocarcinoma & 42 & 17 & 25 & 3.264 & 0.515 \\
\hline Papillary adenocarcinoma & 3 & 0 & 3 & & \\
\hline Mucous adenocarcinoma & 3 & 2 & 1 & & \\
\hline Signet ring cell carcinoma & 18 & 8 & 10 & & \\
\hline Poorly differentiated adenocarcinoma & 36 & 13 & 23 & & \\
\hline \multicolumn{6}{|l|}{ FGFR4 status } \\
\hline Low expression & 33 & 17 & 16 & 1.083 & 0.394 \\
\hline High expression & 69 & 28 & 41 & & \\
\hline \multicolumn{6}{|l|}{ STAT3 status } \\
\hline Negative & 37 & 14 & 23 & 0.929 & 0.408 \\
\hline Positive & 65 & 31 & 34 & & \\
\hline \multicolumn{6}{|l|}{ p-STAT3 status } \\
\hline Negative & 46 & 27 & 19 & 7.222 & 0.009 \\
\hline Positive & 56 & 18 & 38 & & \\
\hline \multicolumn{6}{|l|}{ Vimentin status } \\
\hline Negative & 41 & 24 & 17 & 5.781 & 0.025 \\
\hline Positive & 61 & 21 & 40 & & \\
\hline \multicolumn{6}{|l|}{ E-cadherin status } \\
\hline Negative & 35 & 12 & 23 & 2.089 & 0.208 \\
\hline Positive & 67 & 33 & 34 & & \\
\hline
\end{tabular}

FGFR4, fibroblast growth factor receptor 4; GC, gastric cancer; WHO, World Health Organization. 

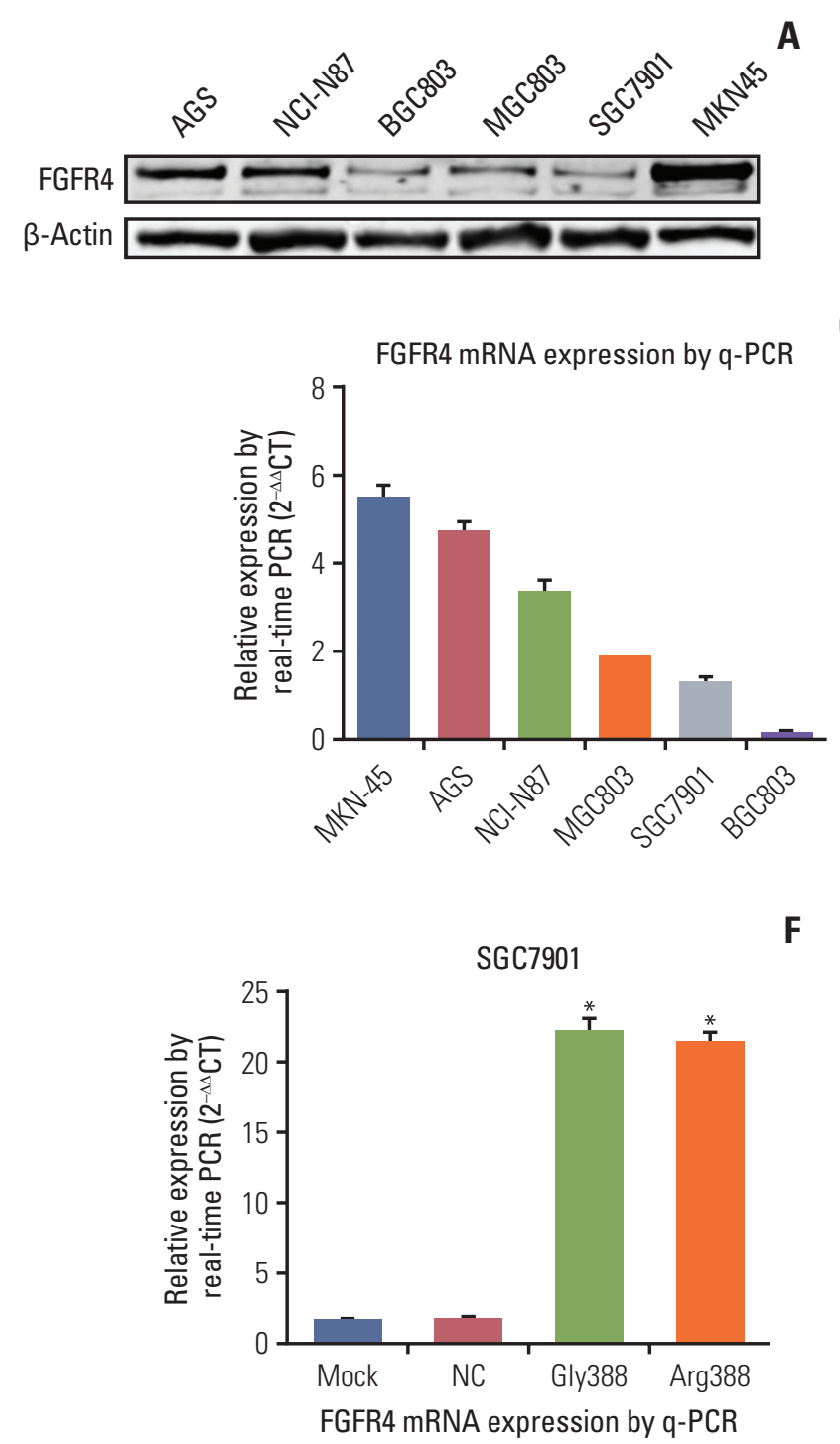

A

$\mathbf{F}$

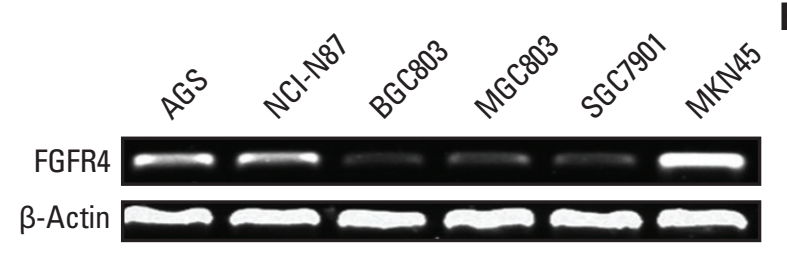

C
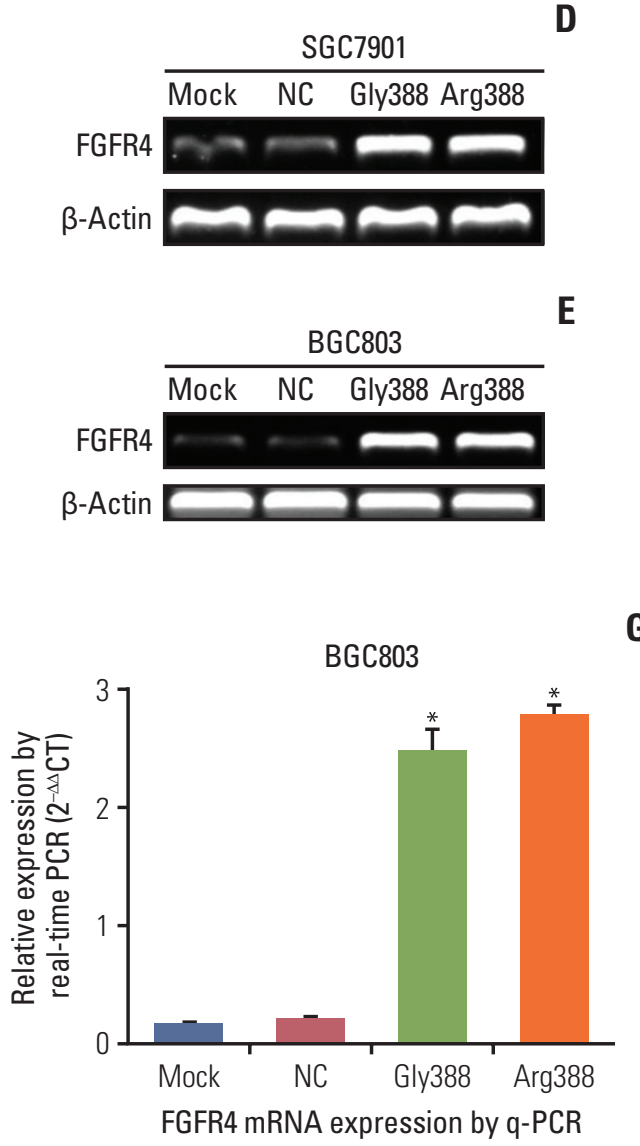

Fig. 2. The expressions of fibroblast growth factor receptor 4 (FGFR4) mRNA and protein were illustrated in various gastric cancer (GC) cell lines and transfected cells. (A) Expressions of FGFR4 protein in different GC cell lines by western blot. (B) Expressions of FGFR4 mRNA in different GC cells by reverse transcription polymerase chain reaction (RT-PCR). (C) Expressions of FGFR4 mRNA in different GC cells by quantitative real-time polymerase chain reaction (q-PCR). Expressions of FGFR4 mRNA in SGC7901 (D) and BGC803 (E) cells (mock, negative control [NC], Gly388-transfected, and Arg388-transfected) by RT-PCR. Expressions of FGFR4 mRNA in SGC7901 (F) and BGC803 (G) cells (mock, NC, Gly388-transfected, and Arg388-transfected) by q-PCR. $\beta$-Actin was served as loading control. At least three independent detecting were performed. * $\mathrm{p}<0.05$.

were calculated by Kaplan-Meier method, and differences between survival curves were examined with the log-rank test. All the statistical tests were bilateral, with a significance at the 0.05 level. SPSS ver. 17.0 statistical software (SPSS Inc., Chicago, IL) was used for statistical analysis and graph drawing, which was used to collect and classify patients' clinical pathology and follow-up data, covering sex, age, tumor size, differentiation, tumor stage, lymph node status, distant metastases, Borrmann type (divided into local type including early GC, uplift type [I], ulcer type [II] and infiltrating type including infiltrating ulcer type [III] and diffuse infiltrating type [IV]), Lauren type, World Health Organization type (WHO), and expression of relevant tumor markers.

\section{Ethical statement}

The study was approved by the Institutional Review Board of The First Affiliated Hospital of Zhengzhou University (IRB No. YB M-05-02) and performed in accordance with the principles of the Declaration of Helsinki. Written informed consents were obtained. 


\section{Results}

1. Correlation of FGFR4 Gly388Arg single nucleotide polymorphism with clinicopathological characteristics and prognosis of GC patients

The relationship between pathological parameters and related protein expression in 102 GC patients and FGFG4 polymorphism was summarized in Table 1. We then stratified FGFR4-Gly388 and FGFR4-Arg388 patients according to FGFR4 single nucleotide polymorphism (SNP). FGFR4Arg388 variant was observed in GC patients with higher tumor stage $(\mathrm{T} 3+\mathrm{T} 4)(\mathrm{p}=0.047)$, while no correlation with sex $(p=0.373)$, age $(p>0.99)$, tumor size $(p>0.99)$, differentiation $(\mathrm{p}=0.227)$, lymph node status $(\mathrm{p}=0.436)$, distant metastases $(\mathrm{p}=0.130)$, Borrmann type $(\mathrm{p}=0.071)$, Lauren type $(\mathrm{p}=0.840)$, and WHO type $(p=0.515)$. It was worth mentioning that Borrmann type $(\mathrm{p}=0.071)$ may be statistically significant if the sample size was increased. A significant correlation was observed between FGFR4-Arg388 variant and the expression of $\operatorname{Vim}(p=0.025)$ as well as $p-S T A T 3(p=0.009)$ expression in GC, while no correlation with the expression of FGFR4 $(p=0.394)$, STAT3 ( $p=0.408)$, and E-cad $(p=0.208)$. Moreover, Kaplan-Meier survival analysis showed that FGFR4-Arg388 variant in GC patients were significantly correlated with a poorer prognosis in terms of cumulative survival compared with FGFR4-Gly388 allele ( $\mathrm{p}=0.033$ ) (Fig. 1B). Typical immunohistochemical positive expression of the five related molecules and H\&E staining for morphological features of GC can be seen in Fig. 1C.

\section{FGFR4 expression differed in various GC cell lines}

FGFR4 was expressed in GC lines at mRNA and protein levels using q-PCR, reverse transcription PCR (RT-PCR), and western blot analysis. As Fig. 2A displayed, the protein expression of FGFR4 was more obvious in MKN45 and AGS, while weaker in MGC803, SGC7901, and BGC803. RT-PCR results showed that mRNA expression of FGFR4 was distinctly stronger in MKN45 and AGS than in the other four GC cell lines (Fig. 2B). As showed in Fig. 2C, the quantitative analysis results by q-PCR verified that the mRNA expression of FGFR4 in SGC7901 and BGC803 was weaker than the other GC cell lines. Hence, the SGC7901 and BGC803 cell lines which were utilized to construct different FGFR4 genetic phenotype were chosen to conduct subsequent assays.

\section{FGFR4-Gly388 allele and FGFR4-Arg388 allele were veri- fied to be over-expressed}

The stable overexpression of FGFR4-Gly388 and FGFR4Arg388 in GC cells were conducted by transfection of lentiviral overexpression vector (pHBLV-CMV-MCS-3flag-EF1puro) for the following functional assays. The efficiency of transfection of vector was checked by RT-PCR and q-PCR. As Fig. 2D and E shown, the expression of FGFR4-Gly388 and
FGFR4-Arg388 mRNA was remarkably increased in SGC7901 and BGC803 (vector transfection groups) compare with mock and NC groups. As shown in Fig. 2F and G, the quantitative analysis results by q-PCR also verified that FGFR4 mRNA expression in SGC7901 and BGC803 vector transfection groups was significantly higher. Actually, all tests had proved effective in increasing FGFR4 mRNA expression, as measured by RT-PCR and q-PCR.

\section{FGFR4-Arg388 allele enhanced proliferation, invasion, and migration}

Compared with mock and NC cells, the proliferation of Gly388-transfected and Arg388-transfected cells was considerably enhanced. Results of CCK-8 proliferation assay showed that the absorbency was higher in FGFR4-Arg388 groups than that in FGFR4-Gly388 groups, particularly after 3, 4, and 5 days of culture (Student's t test, $\mathrm{p}<0.05$ ) (Fig. 3A). Similarly, proliferation of BGC803 Arg388-tranfected cells obviously increased in comparison to the other three groups $(\mathrm{p}<0.05)$ (Fig. 3B). The same results were achieved for EdU proliferation assay. The proportion of Arg388-transfected cells proliferation were higher compared to the other three groups in SGC7901 (Students t test, $\mathrm{p}<0.05$ ) (Fig. 3C and E) and BGC803 (Student's t test, p < 0.05) (Fig. 3D and F). When compared with mock and NC groups, Gly388-transfected and Arg388-transfected cells showed significantly more cell invasion. As shown in Fig. 3G, the number of SGC7901 cells invading the Matrigel-coated membrane in the FGFR4-Arg388 group was much higher than that in the FGFR4-Gly388 group ( $p<0.05$ ) (Fig. 3I). Similarly, the number of BGC803 cells in the FGFR4-Arg388 group was significantly higher than that in the FGFR4-Gly388 group (Fig. 3H), and the difference was statistically significant $(p<0.05)$ (Fig. 3J). Similar results were also observed using a cell scratch assay in SGC7901 (Fig. 3K and M) and BGC803 ( $<$ < 0.05) (Fig. 3L and N). Together, these results indicated that both the Arg388-transfected and Gly388-transfected cells evidently increased the proliferation, migration, and invasion in GC cells, whereas that overexpression of the FGFR4-Arg388 variant was stronger than Gly388 allele.

\section{FGFR4-Arg388 variant can increase the resistance of OXA}

To assess the effect of OXA on the growth of SGC7901 and BGC803 cells at different concentrations, we used CCK-8 proliferation assay to observe cell viability. Fig. 4A and B show the cell viability of SGC7901 and BGC803 cells at different concentrations of OXA, and the $\mathrm{IC}_{50}$ in Arg388-transfected, Gly388-transfected, mock and NC were 12.83, 10.46, 5.77, and $5.12 \mu \mathrm{g}$, respectively (Fig. $4 \mathrm{~A}$ and $\mathrm{B}$ ). When OXA was treated BGC803 cells, the cell viability also decreased with increasing concentration. The $\mathrm{IC}_{50}$ in Arg388-transfected, Gly388-transfected, mock, and NC were 11.67, 6.86, 1.96, and 

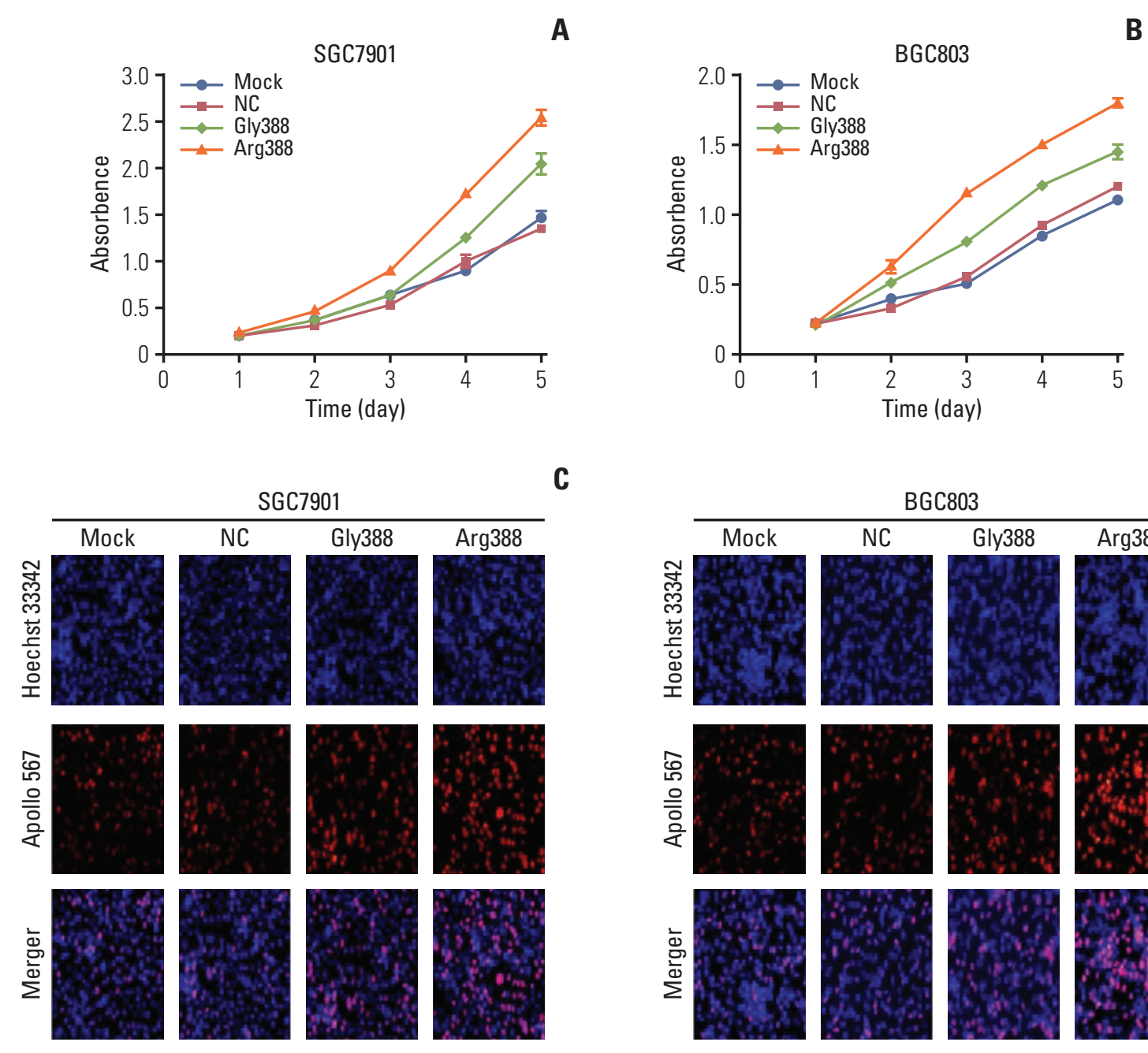

C
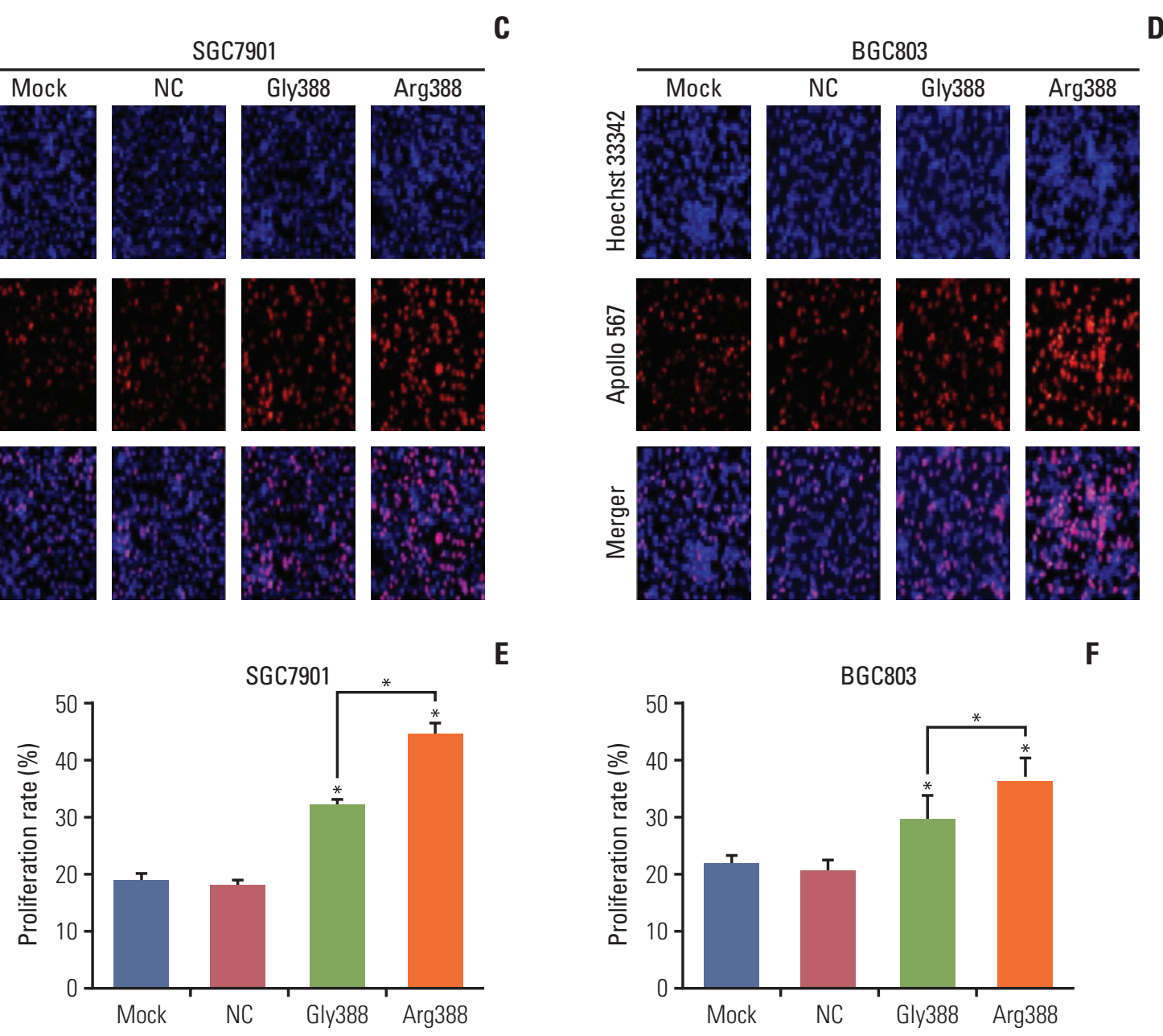

Fig. 3. Effect of fibroblast growth factor receptor 4 (FGFR4) genotype on proliferation, invasion, and migration. (A, B) Compared with mock and negative control (NC) cells, the proliferation of Gly388- and Arg388-transfected cells was significantly enhanced. In addition, Arg388-transfected cells have a faster proliferation than Gly388-transfected cells. In addition, Arg388-transfected cells have a faster proliferation than Gly388-transfected cells. Similar results were also observed in the EdU fluorescence staining test (C-F), Transwell chambers invasion assay (G-J), and cell scratch assay $(\mathrm{K}-\mathrm{N}) .{ }^{*} \mathrm{p}<0.05$. (Continued to the next page) 
G
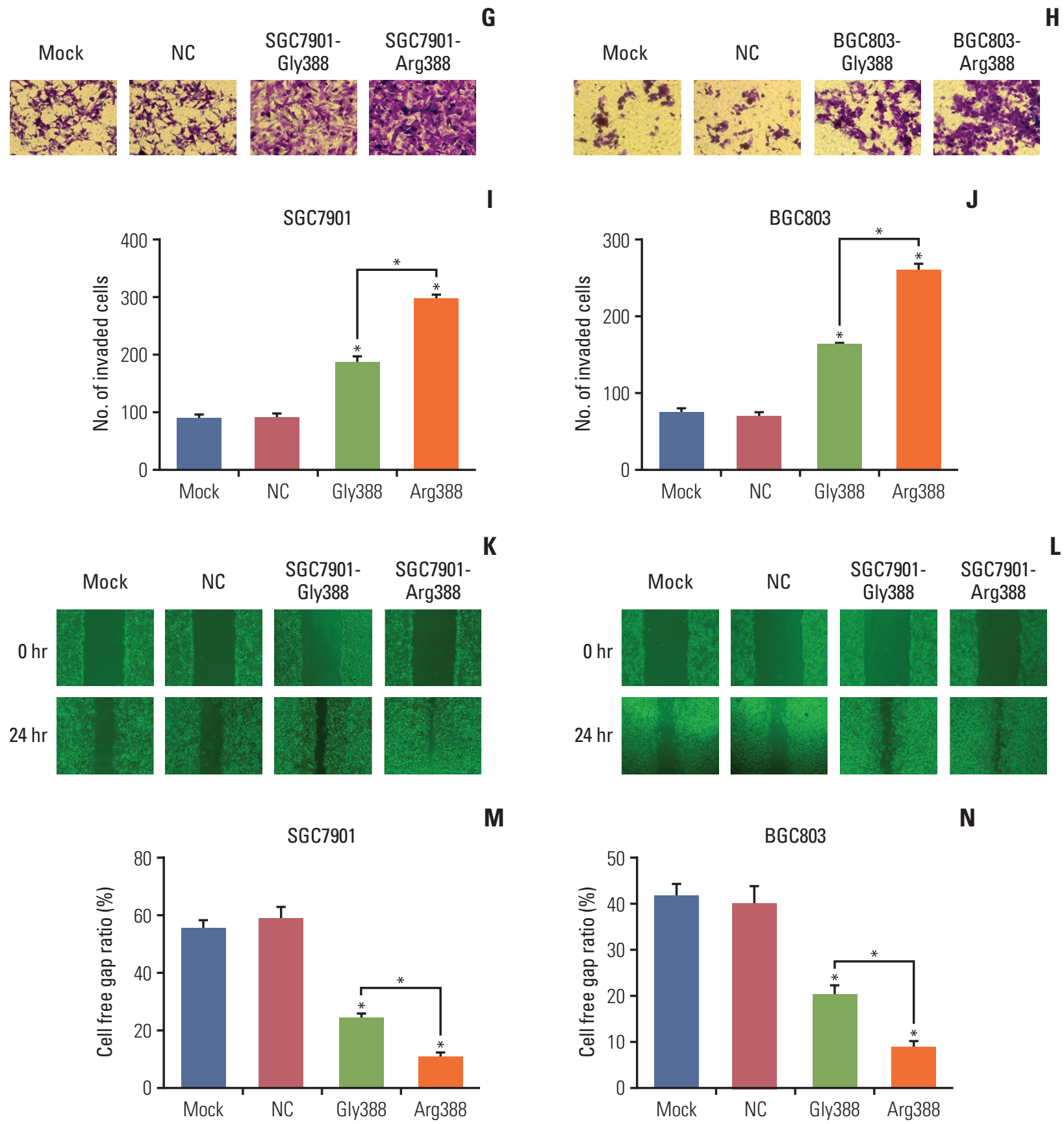

Fig. 3. (Continued from the previous page) Similar results were also observed in the EdU fluorescence staining test (C-F), Transwell chambers invasion assay (G-J), and cell scratch assay $(\mathrm{K}-\mathrm{N}) .{ }^{*} \mathrm{p}<0.05$.

$2.27 \mu \mathrm{g}$, respectively (Fig. $4 \mathrm{C}$ and D). Based on the above results, the appropriate concentration of OXA for later apoptosis experiments was $5 \mu \mathrm{g}$ in SGC7901 cells and $2 \mu \mathrm{g}$ in BGC803 cells, respectively (average value of $\mathrm{IC}_{50}$ for mock and NC group).

In cell apoptosis flow cytometry assay, when compared to mock and NC group, the Gly388- and Arg388-transfected group distinctly reduced the apoptosis rate of SGC7901 cells (Student's t test, $p<0.05$ ) (Fig. 4E and G) and BGC803 cells (Student's t test, $\mathrm{p}<0.05$ ) (Fig. $4 \mathrm{~F}$ and $\mathrm{H}$ ). Moreover, the apoptosis rate of Arg388-transfected group was less than that of Gly388-transfected group in both of these GC cells. Furthermore, similar results could be shown in fluorescent staining for apoptosis (Fig. 4I-L), which suggested that FGFR4 overexpression can weaken the chemotherapy effect of OXA, and it was important to note that the FGFR4-Arg388 variant was superior to the FGFR4-Gly388 allele to increase the resistance of OXA. 

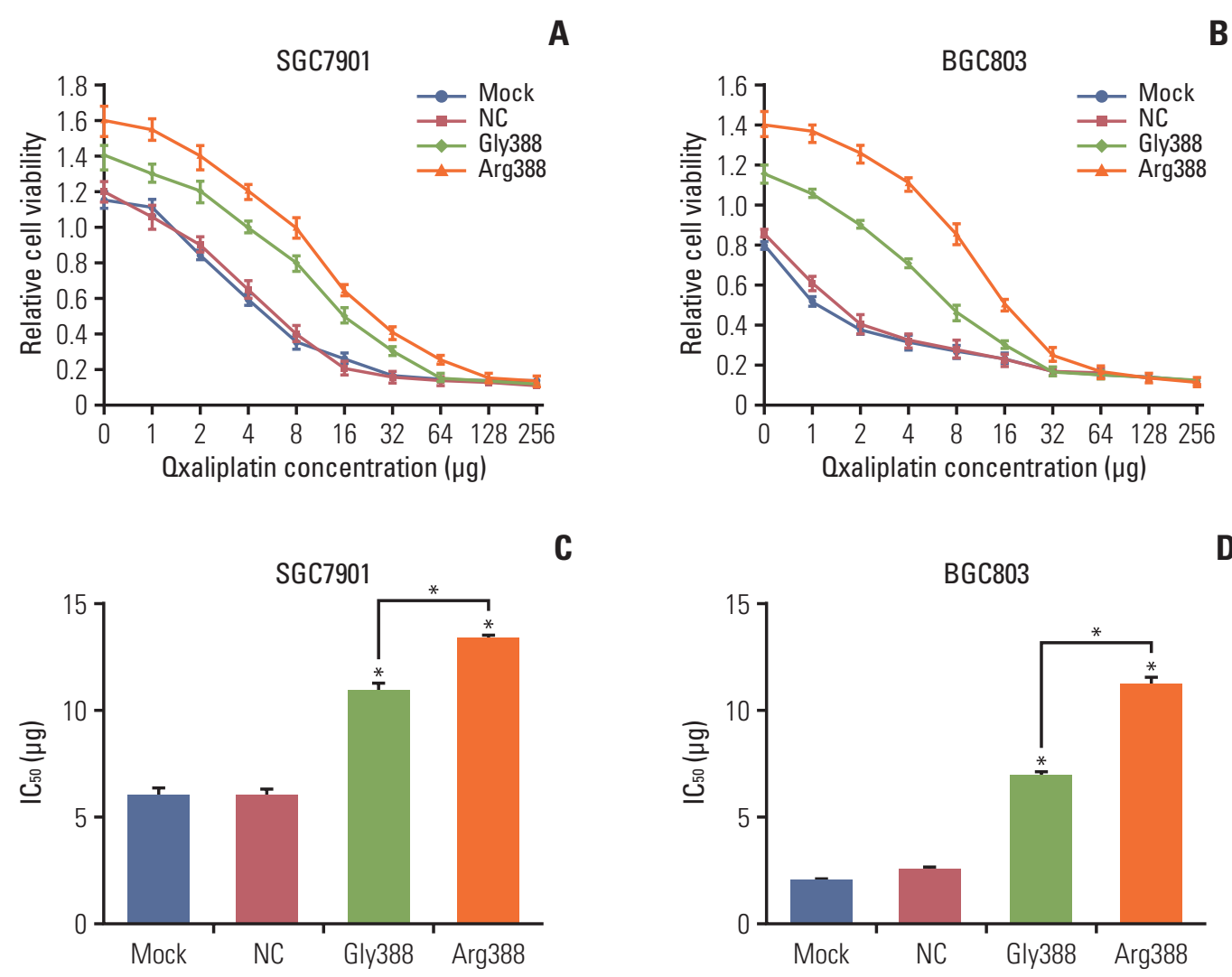

Fig. 4. The relationship between fibroblast growth factor receptor 4 (FGFR4) genotype and oxaliplatin (OXA) resistance. (A-D) Effects of different OXA concentrations on the activity of SGC7901 and BGC803 cells. NC, normal control. (Continued to the next page)

After 24 hours of OXA culture, expressions of caspase-3 and cleaved caspase-3 increased substantially in SGC7901 cells. Compared with mock, NC, and Gly388-transfected groups, the Arg388-transfected group decreased the expressions of caspase- 3 and cleaved caspase- 3 , whether adding OXA or not, which confirmed that the apoptosis rate of FGFR4Arg388 variant group was lower than the other three groups (Fig. 4M). Moreover, similar results could be observed in BGC803 cells (Fig. 4N).

\section{FGFR4-Arg388 variant affects the EMT process and STAT3 signaling pathway, and inhibition of the STAT3 pathway reduces Arg 388 variant induced EMT}

To investigate the mechanism of how FGFR4-Arg388 variant affected the proliferation, invasion, and apoptosis of GC cells, we observed the expression change of associated molecules through western blot. Compared with mock and NC groups, the expression of FGFR4 in the Gly388- and Arg388-transfected SGC7901 cells were equally increased, which were actually shown to be effective in increasing FGFR4 protein expression in transfected GC cells. When the relationship between FGFR4 genotype and STAT3 signaling pathway was studied, the expression of STAT3 was obviously increased in Gly388- and Arg388-transfected SGC7901 cells. The expression of p-STAT3 was substantially increased in Arg388-transfected SGC7901 cells when compared with other three groups. This indicates that the FGFR4-Arg388 variant affects STAT3 signaling pathway. To study the role of the FGFR4 genotype in the EMT, we assessed the roles of the FGFR4-Arg388 variant compared with the FGFR4-Gly388 allele during induction of EMT changes in stably transfected GC cells. Western blot analyses showed there was an increased expression of Vim in Arg388-transfected SGC7901 cells compared with Gly388-transfected and control cells. While E-cad was significantly reduced in Arg388-transfected SGC7901 cells when compared with other three groups. These results implied that the FGFR4-Arg388 variant promotes the EMT process (Fig. 5A). Similar results were also found in BGC803 cells (Fig. 5B).

To explore if FGFR4-Arg388 induced EMT could be decreased by inhibition of STAT3 activation, we cultivated the GC cells with the Jak2 inhibitor AG490 to block STAT3 activation and reduced expression of STAT3 protein. STAT3 and p-STAT3 have the same molecular weight, the expression of two proteins cannot be displayed in one PVDF membrane, that is why we used two internal reference proteins as a control for loading. STAT3 pathway inhibition was followed by growth of E-cad and reduction of Vim both 


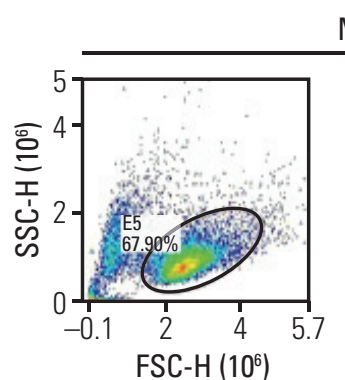

Mock
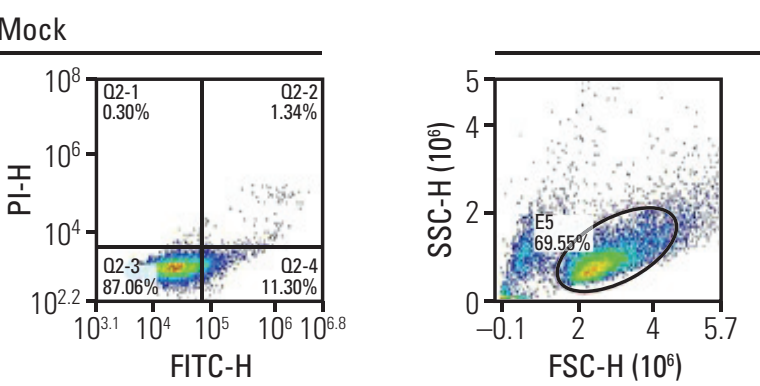

NC

E

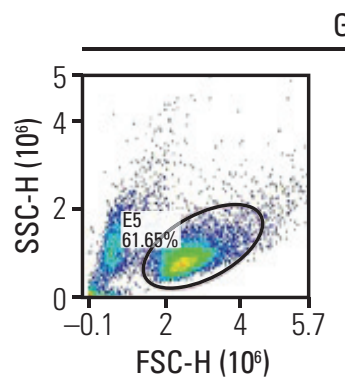

Gly388
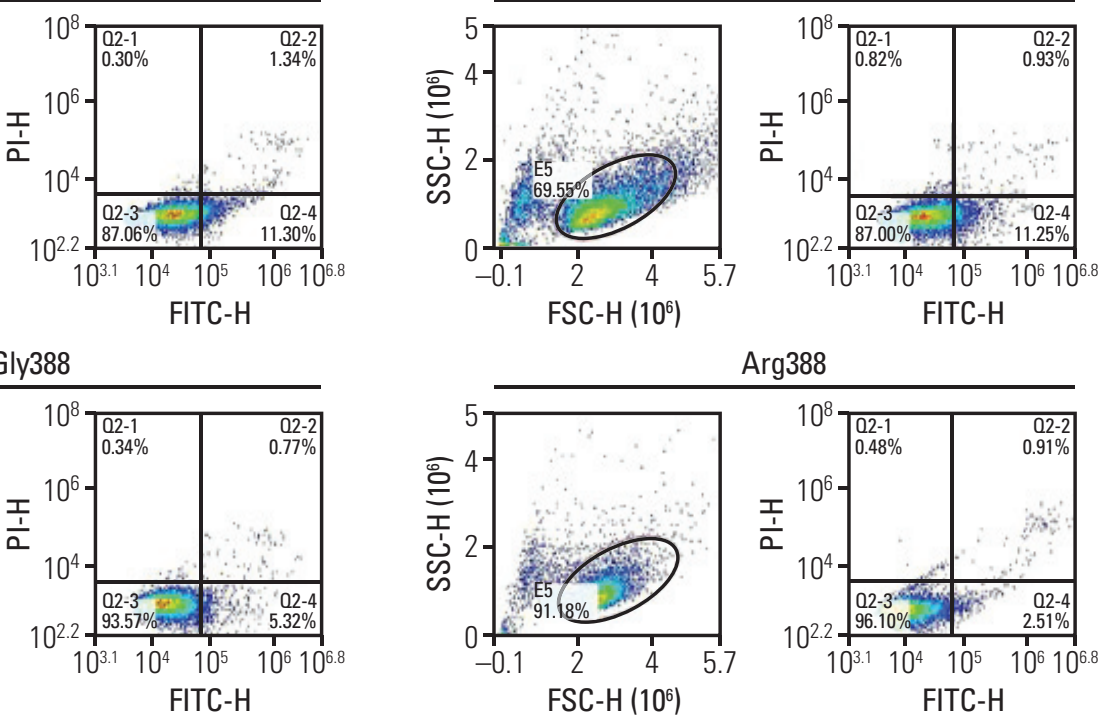

Arg388
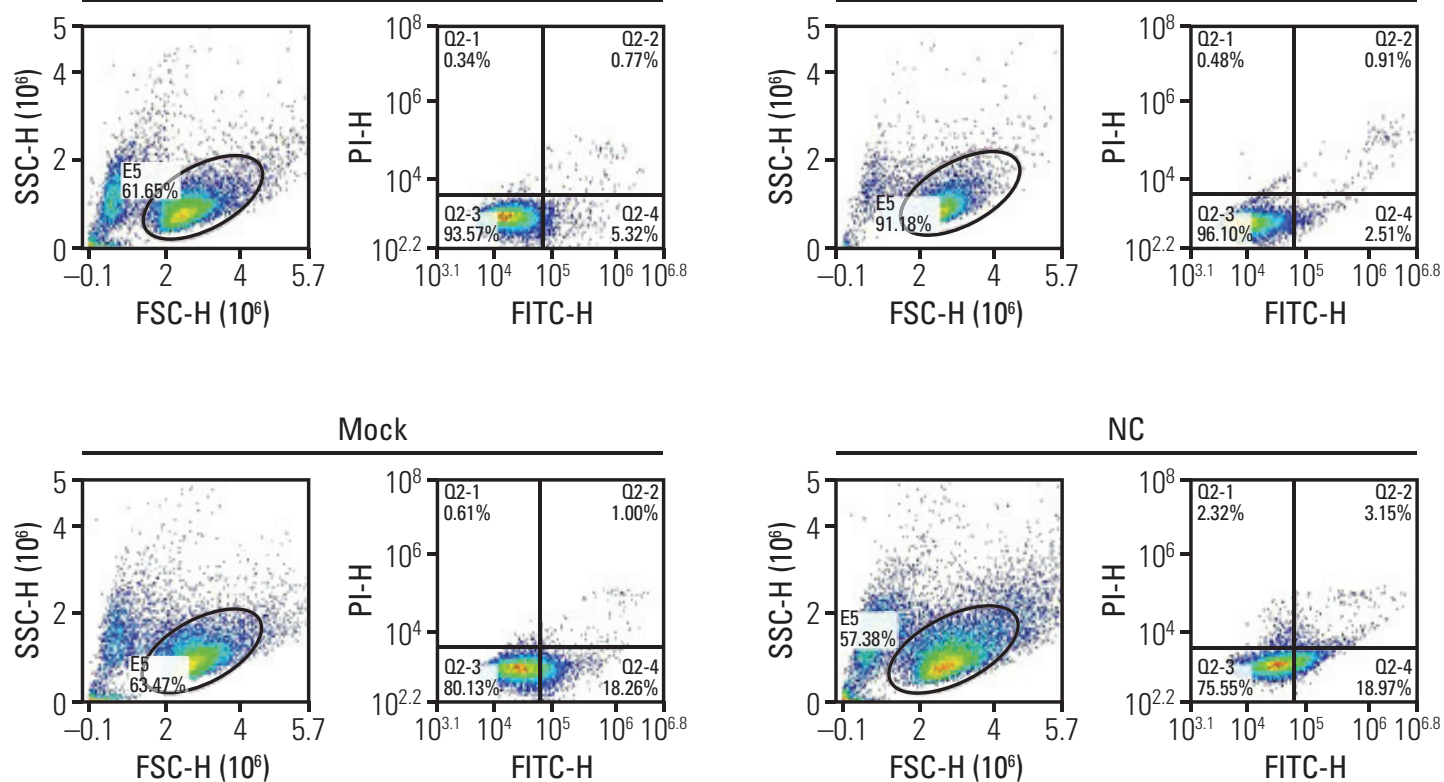

Mock
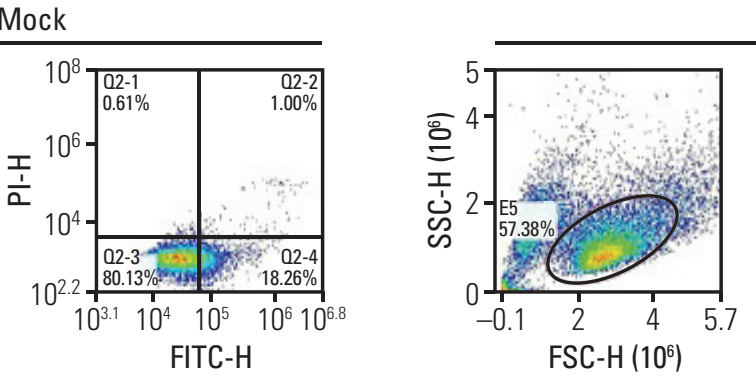

$\mathrm{NC}$
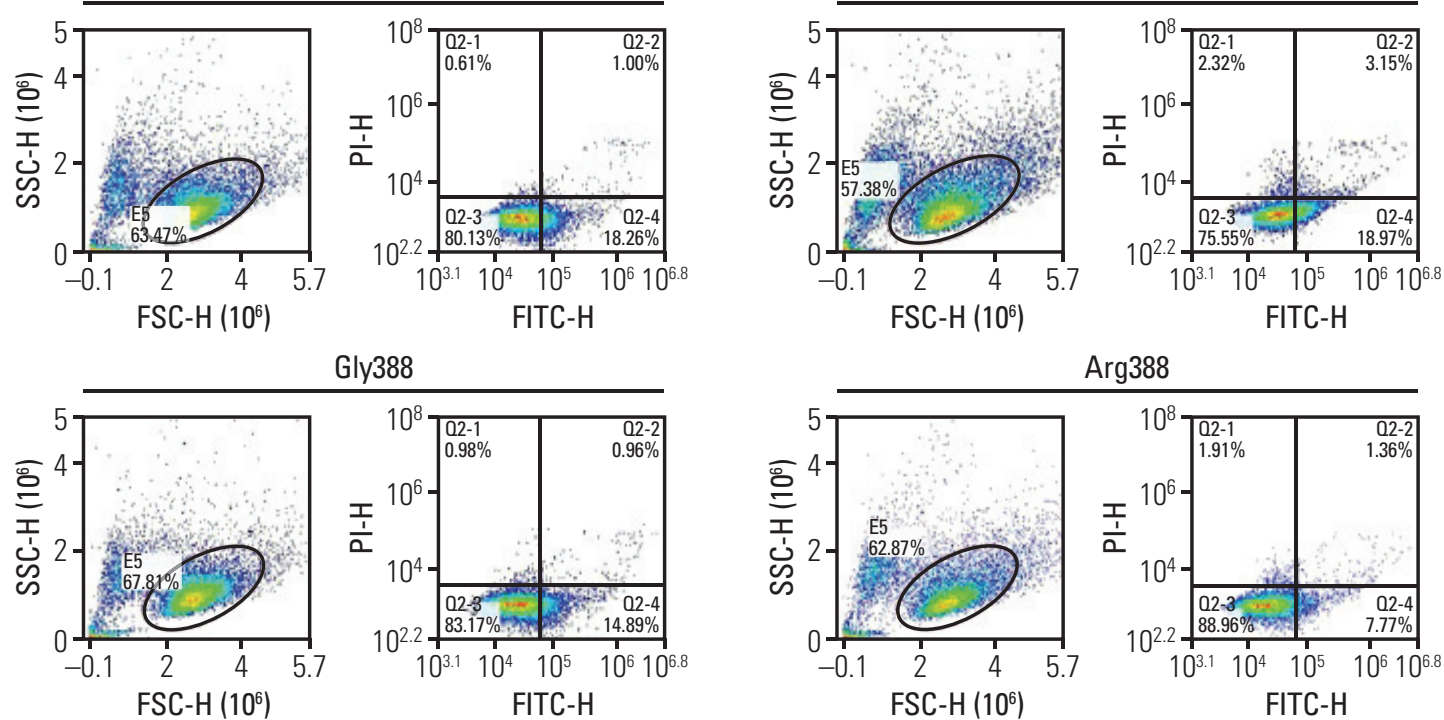

Gly388
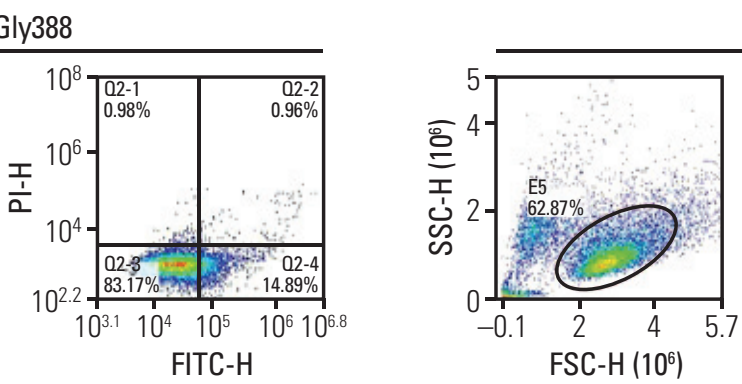

Arg388
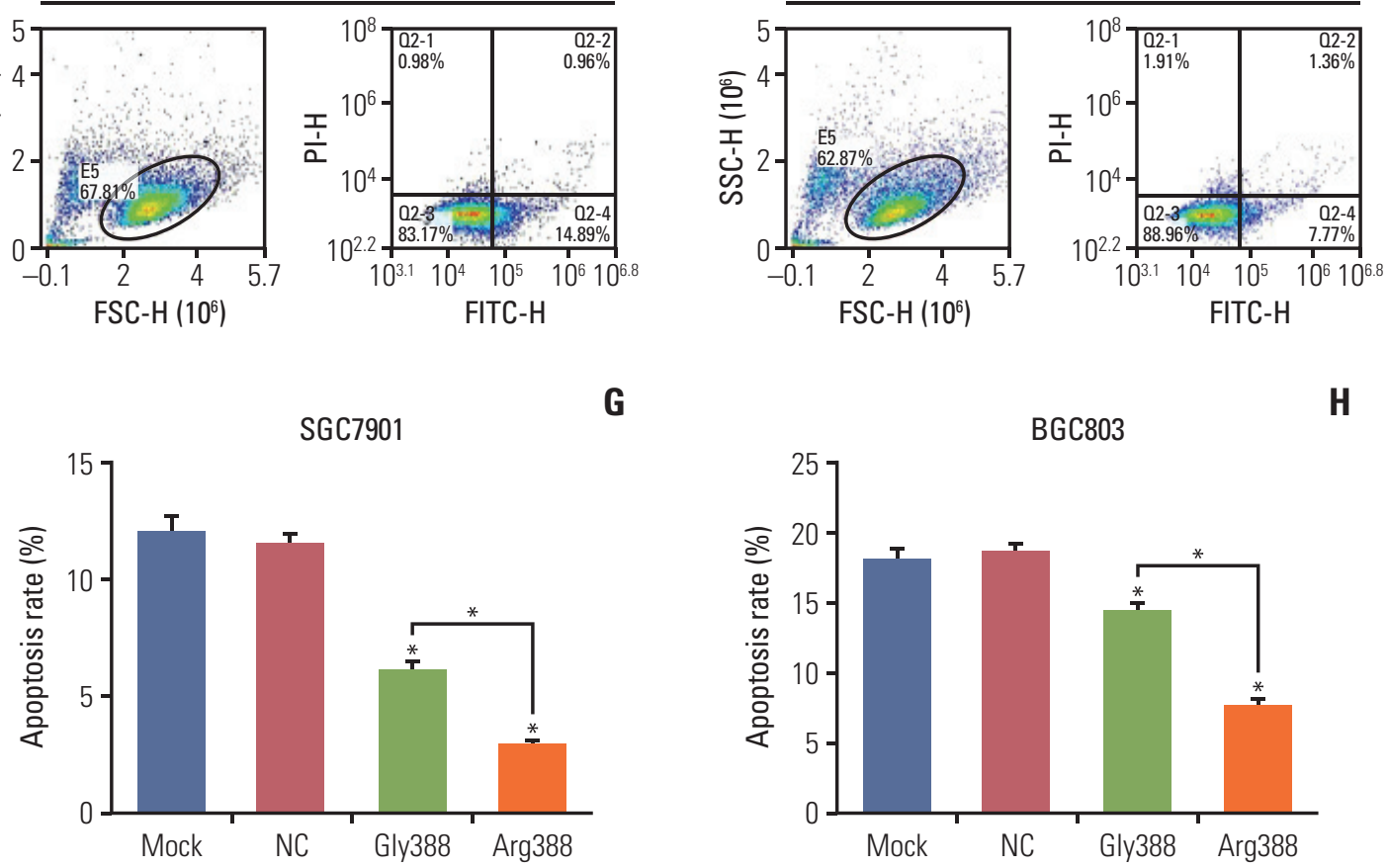

G

BGC803

H

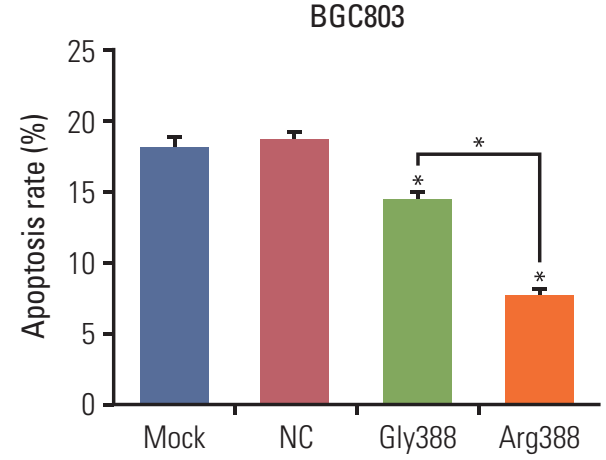

Fig. 4. (Continued from the previous page) (E-H) Flow cytometry: apoptosis rate of gastric cancer (GC) cells with different FGFR4 genotypes after OXA addition for 24 hours. (Continued to the next page) 

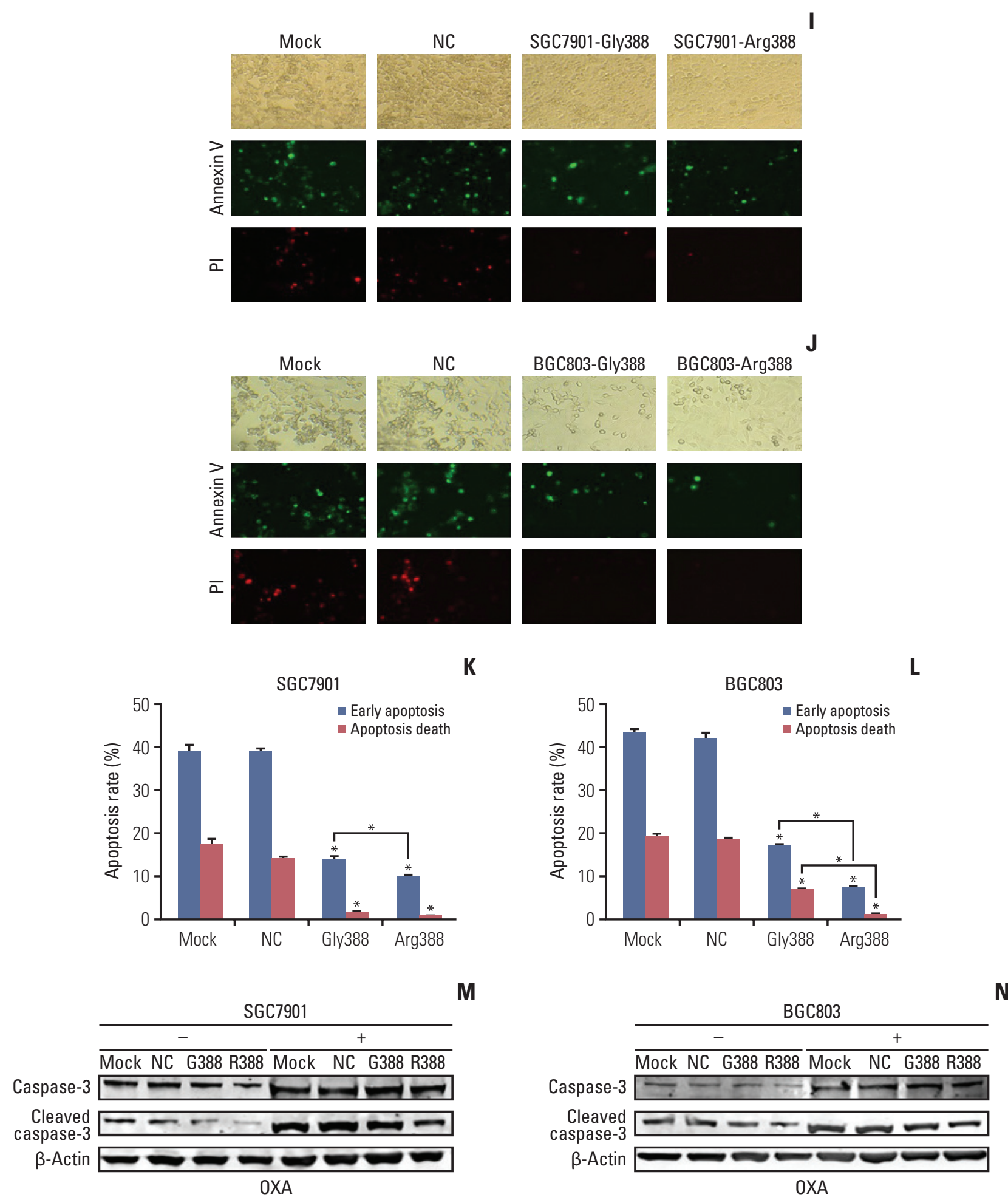

Fig. 4. (Continued from the previous page) (I-L) Fluorescent staining for apoptosis of GC cells with different FGFR4 genotypes (green represents early apoptosis and red represents apoptosis death). PI, propidium iodide. (M, N) Western bolt was used to detect the changes of apoptosis molecules in GC cells with different FGFR4 genotypes before and after OXA addition. ${ }^{*} \mathrm{p}<0.05$. 

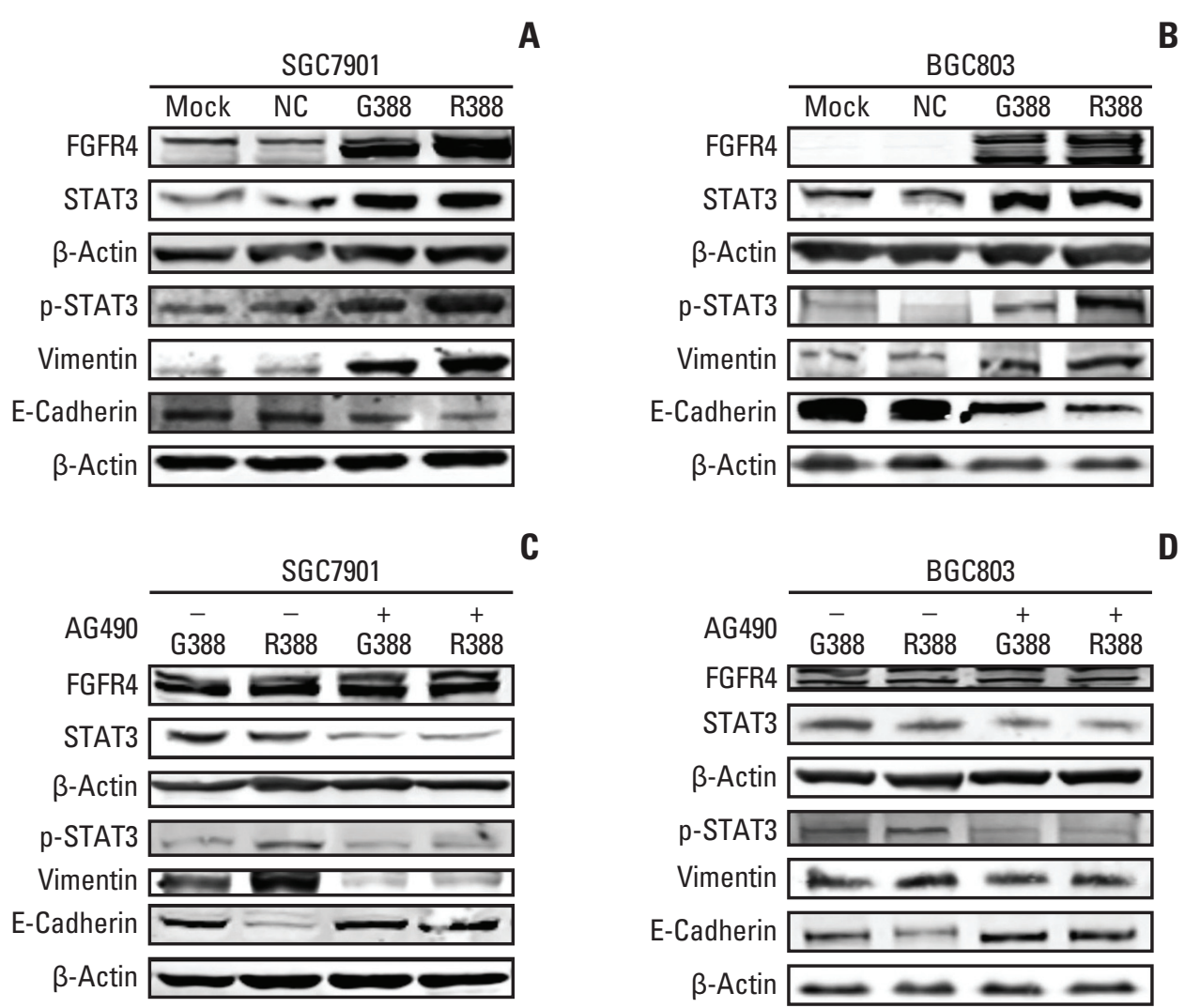

Fig. 5. The mechanism of fibroblast growth factor receptor 4 (FGFR4)-Arg388 influencing the oncogenic properties of gastric cancer cells. (A, B) Western blot detection of the expression of related molecules including FGFR4, signal pathway (STAT3), and epithelial to mesenchymal transition (E-cadherin and vimentin) in SGC7901 and BGC803 cells with different FGFR4 genotypes (mock, normal control [NC], Gly388, and Arg388). (C, D) The changes of related molecules between overexpression Gly388- and Arg388-transfected cells were detected, when cells were incubated with the Jak2 inhibitor AG490 to block STAT3 activation.

in Gly388- and Arg388-transfected cells, while the effect of inhibiting EMT on Arg388 variant was significantly stronger than that of Gly388 allele (Fig. 5C). Notably, similar results were shown in BGC803 cells (Fig. 4D). These results confirmed that FGFR4-Arg388 induced EMT could be reduced by STAT3 pathway obstruction.

\section{Discussion}

The presence of the FGFR4-Arg388 variant correlated with higher tumor stage $(\mathrm{T} 3+\mathrm{T} 4)$ in clinical GC samples $(\mathrm{p}=0.047)$. The results indicated that the FGFR4-Arg388 variant might accelerate the progression of GC. Serra et al. [22] discovered that FGFR4-Arg388 variant promoted tumor progression by increasing peritoneal spread and the growth of metastatic lesions in the liver in the pancreatic endocrine tumor mouse model transfected with FGFR4-Gly388 or Arg388. This may be one of the reasons that the FGFR4-Arg388 variant influenced the progression of GC. Our research found that the overall survival (OS) of GC patients with FGFR4-Arg388 variant was worse than that of FGFR4-Gly388 patients ( $\mathrm{p}=0.033$ ). Quintanal-Villalonga et al. $[16,23]$. indicated that the presence of the FGFR4-Arg388 variant is associated with poorer prognosis in clinical non-small cell lung cancer samples and lung squamous cell carcinoma samples. Multivariate analysis supported the independent prognostic role of the FGFR4-Arg388 variant in OS $[16,23]$. Similar results were found FGFR4-Arg388 variant had a predictive role in the response of esophageal cancer patients to chemoradiotherapy with a worse trend for OS than Gly388 in the early stages [24]. Immunohistochemistry results demonstrated that the expression of Vim, STAT3, and p-STAT3 in FGFR4-Arg388 group was significantly higher than that in Gly388 allele, suggesting that the biological behavior was more aggressively in GC patients with high expression of FGFR4-Arg388, who might be suitable to undergo the chemotherapy after surgery. This may be an explanation that tumor stage was higher and OS was lower in GC patients with FGFR4-Arg388 comparing to that in Gly388 group.

Our previous studies have found that overexpression of FGFR4 continued to increase the infiltration and prolifera- 
tion capacity of GC cells [2]. More importantly, in the present study we focus on comparing the differences in tumor carcinogenic behavior between the two types of overexpression FGFR4 genotypes. Our function assays in vitro revealed that the proliferation, invasion and migration abilities of SGC7901 and BGC803 cells with FGFR4-Arg388 were much stronger than those in FGFR4-Gly388 cells, indicating that the FGFR4 might increase the malignancy of GC cells, which confirmed once again the previous findings. FGFR4 gene could accelerate progression of GC [2]. Similar results were discovered in colorectal cancer by Cho et al. [15].

The proliferation ability of SGC7901 and BGC803 cells with FGFR4-Gly388, Arg388, mock, and NC was significantly weakened when dealt with by different concentration of OXA. Transfection groups had significantly higher $\mathrm{IC}_{50}$ than the mock and NC groups, and the Arg388-transfected group had a higher $\mathrm{IC}_{50}$ than Gly388-transfected group, which suggested that overexpression of FGFR4 was resistant to OXA, whereas FGFR4-Arg388 variant was more effective. This conclusion was confirmed by the apoptosis assay that the apoptosis rates of Arg388-transfected group were markedly decreased in SGC7901 and BGC803 cells treated with the single concentration of OXA compared with others. In molecular level, the expression of caspase-3 and cleaved caspase-3 obviously weakened in Arg388 group treated with OXA, which supported the results of apoptosis assay. Our former studies have found that FGFR4 overexpression can weaken the effects of 5-fluorouracil, BGJ398 (an inhibitor of FGFR4), and PD173074 (an inhibitor of FGFR4) [2,3]. This study reconfirms the role of FGFR4 to weaken the effects of OXA, a novel clinical chemotherapy drug. Gao et al. [25] showed that inhibition of FGFR4 signaling significantly overcomes sorafenib resistance in hepatocellular carcinoma. Serra et al. [22] showed that unlike FGFR4-Gly388, FGFR4-Arg388 tumors exhibited diminished responsiveness to everolimus (an inhibitor of mammalian target of rapamycin signal). Few studies have been conducted on resistance of FGFR4 Gly388Arg polymorphisms to chemotherapeutic drugs, while we found that FGFR4-Arg388 variant was indeed resistant to OXA compared with Gly388 allele.

Studies have revealed that the FGFR4-Arg388 variant can induce EMT $[15,16]$, activating the STAT3 signaling pathway by promoting phosphorylation of STAT3 [13]. Therefore, we explored the correlation between FGFR4 Gly388Arg polymorphism and EMT as well as STAT3 signaling pathways in GC. The results of western blot showed the expression of STAT3 was obviously increased in Gly388- and Arg388-transfected cells. The expression of p-STAT3 was substantially increased in Arg388-transfected cells when compared with other three groups, while there was no difference in expression of STAT3 between Gly388- and Arg388-transfected groups. Immunohistochemical results showed that the expressions of p-STAT3 were relatively higher in Arg388 GC pati- ents, which were in accordance with western bolt results. This suggests that the FGFR4-Arg388 variant affects the STAT3 signaling pathway, possibly increasing the phosphorylation of STAT3. Ezzat et al. [6] showed that FGFR4-Arg388 pituitary cells have higher mitochondrial STAT3 serine phosphorylation driving basal and maximal oxygen consumption rate than ones expressing the more common FGFR4Gly388 allele. This phenomenon may be ascribed to that FGFR4-Arg388 can expose a proximal STAT3 binding site [12]. A large number of studies have found STAT3 activation was involved in regulating expression of apoptosis genes, yet continuous activation of STAT3 signaling would grant resistance to apoptosis in breast cancer cells [26,27]. FGFR4Arg388 variant could activate STAT3 signal, which may be the reason why Arg388 had the least apoptosis of GC cells treated with OXA.

Western bolt showed there was a higher expression of Vim and lower expression of E-cad in FGFR4-Arg388 group, which suggested that Arg388 variant may induce EMT. Elevated Vim protein levels and reduced E-cad protein levels are usually associated with cancer cell metastasis and the EMT process. Cho et al. [15] had similar findings in colorectal cancer that FGFR4-Arg388 could induce EMT. Quintanal-Villalonga et al. [16] found that the poorer prognostic role of this FGFR4-Arg388 variant in lung cancer may be mediated by the induction of $\mathrm{N}$-cadherin expression (an EMT molecular). The relationship between FGFR4-Arg388 and E-cad showed differences in immunohistochemistry and western blot results, possibly due to the small number of clinical cases leading to false positive or negative results in immunohistochemistry.

Plenty of studies have found that constitutive STAT3 expression promoted and sustained the EMT phenotype [28-30]. Meanwhile, we found that FGFR4-Arg388 was substantially correlated with the molecules of EMT and STAT3 signal. Thus, we proposed a hypothesis that FGFR4-Arg388, STAT3, and EMT make up a functional signal axis that regulates carcinogenesis of GC and inhibition of FGFR4-Arg388/ STAT3/EMT signal axis was possible to reverse the EMT. After final verification, the activation of STAT3 inhibited by AG490 increased the expression of E-cad and decreased the expression of Vim, indicating that the inhibition of STAT3 attenuated the process of EMT. Finally, it can be understood that blocking this signal axis can reverse the EMT process. We also found AG490 inhibits overexpression of FGFR4-Arg388 variant induced EMT more significantly than Gly388 allele, which could be due to FGFR4-Arg388 promotes STAT3 phosphorylation more effectively [6,12]. It is worth further explore the exact mechanisms involved FGFR4-Arg388/STAT3 / EMT regulating the biological function of tumor cells.

Collectively, this study elucidated the role of FGFR4Arg388 variant in GC, and emphasized the probability of this SNP as potential therapeutic targets. FGFR4-Arg388 variant 
was linked to poor prognosis of GC patients. FGFR4-Arg388 variant increased proliferation and invasion GC cells, weakening the effects of OXA. The exploration mechanism may be through FGFR4-Arg388/STAT3/EMT axis regulating pivotal oncogenic properties of GC cells.

\section{Conflict of Interest}

Conflicts of interest relevant to this article was not reported.

\section{Acknowledgments}

This study was supported by Department of Gastrointestinal Surgery and Institute of Clinical Medicine, The First Affiliated Hospital, Zhengzhou University and National Natural Science Foundation of China, Grant No. 81201955. Furthermore, this work was supported by the Foundation of Henan Educational Committee (Grant No.19A320080).

\section{References}

1. Katoh M. Genetic alterations of FGF receptors: an emerging field in clinical cancer diagnostics and therapeutics. Expert Rev Anticancer Ther. 2010;10:1375-9.

2. Li J, Ye Y, Wang M, Lu L, Han C, Zhou Y, et al. The overexpression of FGFR4 could influence the features of gastric cancer cells and inhibit the efficacy of PD173074 and 5-fluorouracil towards gastric cancer. Tumour Biol. 2016;37:6881-91.

3. Ye Y, Jiang D, Li J, Wang M, Han C, Zhang X, et al. Silencing of FGFR4 could influence the biological features of gastric cancer cells and its therapeutic value in gastric cancer. Tumour Biol. 2016;37:3185-95.

4. Ye Y, Shi Y, Zhou Y, Du C, Wang C, Zhan H, et al. The fibroblast growth factor receptor-4 Arg388 allele is associated with gastric cancer progression. Ann Surg Oncol. 2010;17:3354-61.

5. Chou CH, Hsieh MJ, Chuang CY, Lin JT, Yeh CM, Tseng PY, et al. Functional FGFR4 Gly388Arg polymorphism contributes to oral squamous cell carcinoma susceptibility. Oncotarget. 2017;8:96225-38.

6. Ezzat S, Wang R, Pintilie M, Asa SL. FGFR4 polymorphic alleles modulate mitochondrial respiration: a novel target for somatostatin analog action in pituitary tumors. Oncotarget. 2017;8:3481-94

7. Whittle SB, Reyes S, Du M, Gireud M, Zhang L, Woodfield $\mathrm{SE}$, et al. A polymorphism in the FGFR4 gene is associated with risk of neuroblastoma and altered receptor degradation. J Pediatr Hematol Oncol. 2016;38:131-8.

8. Morimoto Y, Ozaki T, Ouchida M, Umehara N, Ohata N, Yoshida A, et al. Single nucleotide polymorphism in fibroblast growth factor receptor 4 at codon 388 is associated with prognosis in high-grade soft tissue sarcoma. Cancer. 2003;98:2245-50.

9. Streit S, Mestel DS, Schmidt M, Ullrich A, Berking C. FGFR4 Arg388 allele correlates with tumour thickness and FGFR4 protein expression with survival of melanoma patients. $\mathrm{Br} \mathrm{J}$ Cancer. 2006;94:1879-86.

10. Yu W, Feng S, Dakhova O, Creighton CJ, Cai Y, Wang J, et al. FGFR-4 Arg(3)(8)(8) enhances prostate cancer progression via extracellular signal-related kinase and serum response factor signaling. Clin Cancer Res. 2011;17:4355-66.

11. Jalali S, Monsalves E, Tateno T, Zadeh G. Role of mTOR inhibitors in growth hormone-producing pituitary adenomas harboring different FGFR4 genotypes. Endocrinology. 2016;157:3577-87.

12. Ulaganathan VK, Ullrich A. Membrane-proximal binding of
STAT3 revealed by cancer-associated receptor variants. Mol Cell Oncol. 2016;3:e1145176.

13. Ezzat S, Zheng L, Florez JC, Stefan N, Mayr T, Hliang MM, et al. The cancer-associated FGFR4-G388R polymorphism enhances pancreatic insulin secretion and modifies the risk of diabetes. Cell Metab. 2013;17:929-40.

14. Sugiyama N, Varjosalo M, Meller P, Lohi J, Chan KM, Zhou Z, et al. FGF receptor-4 (FGFR4) polymorphism acts as an activity switch of a membrane type 1 matrix metalloproteinaseFGFR4 complex. Proc Natl Acad Sci U S A. 2010;107:15786-91.

15. Cho SH, Hong CS, Kim HN, Shin MH, Kim KR, Shim HJ, et al. FGFR4 Arg388 is correlated with poor survival in resected colon cancer promoting epithelial to mesenchymal transition. Cancer Res Treat. 2017;49:766-77.

16. Quintanal-Villalonga A, Ojeda-Marquez L, Marrugal A, Yague P, Ponce-Aix S, Salinas A, et al. The FGFR4-388arg variant promotes lung cancer progression by $\mathrm{N}$-cadherin induction. Sci Rep. 2018;8:2394.

17. Edge SB, Compton CC. The American Joint Committee on Cancer: the 7th edition of the AJCC cancer staging manual and the future of TNM. Ann Surg Oncol. 2010;17:1471-4.

18. Thussbas C, Nahrig J, Streit S, Bange J, Kriner M, Kates R, et al. FGFR4 Arg388 allele is associated with resistance to adjuvant therapy in primary breast cancer. J Clin Oncol. 2006;24:374755.

19. Myoteri D, Dellaportas D, Lykoudis PM, Apostolopoulos A, Marinis A, Zizi-Sermpetzoglou A. Prognostic evaluation of vimentin expression in correlation with Ki67 and CD44 in surgically resected pancreatic ductal adenocarcinoma. Gastroenterol Res Pract. 2017;2017:9207616.

20. Gao H, Lan X, Li S, Xue Y. Relationships of MMP-9, E-cadherin, and VEGF expression with clinicopathological features and response to chemosensitivity in gastric cancer. Tumour Biol. 2017;39:1010428317698368.

21. Zhang S, Huang S, Deng C, Cao Y, Yang J, Chen G, et al. Co-ordinated overexpression of SIRT1 and STAT3 is associated with poor survival outcome in gastric cancer patients. Oncotarget. 2017;8:18848-60.

22. Serra S, Zheng L, Hassan M, Phan AT, Woodhouse LJ, Yao JC, et al. The FGFR4-G388R single-nucleotide polymorphism alters pancreatic neuroendocrine tumor progression and response to mTOR inhibition therapy. Cancer Res. 2012;72: 5683-91.

23. Quintanal-Villalonga A, Carranza-Carranza A, Melendez R, 
Ferrer I, Molina-Pinelo S, Paz-Ares L. Prognostic role of the FGFR4-388Arg variant in lung squamous-cell carcinoma patients with lymph node involvement. Clin Lung Cancer. 2017;18:667-74.

24. Shim HJ, Shin MH, Kim HN, Kim JH, Hwang JE, Bae WK, et al. The prognostic significance of FGFR4 Gly388 polymorphism in esophageal squamous cell carcinoma after concurrent chemoradiotherapy. Cancer Res Treat. 2016;48:71-9.

25. Gao L, Wang X, Tang Y, Huang S, Hu CA, Teng Y. FGF19/ FGFR4 signaling contributes to the resistance of hepatocellular carcinoma to sorafenib. J Exp Clin Cancer Res. 2017;36:8.

26. Calo V, Migliavacca M, Bazan V, Macaluso M, Buscemi M, Gebbia N, et al. STAT proteins: from normal control of cellular events to tumorigenesis. J Cell Physiol. 2003;197:157-68.

27. Okabe M, Miyabe S, Nagatsuka H, Terada A, Hanai N, Yokoi
M, et al. MECT1-MAML2 fusion transcript defines a favorable subset of mucoepidermoid carcinoma. Clin Cancer Res. 2006;12:3902-7.

28. Sullivan NJ, Sasser AK, Axel AE, Vesuna F, Raman V, Ramirez N, et al. Interleukin-6 induces an epithelial-mesenchymal transition phenotype in human breast cancer cells. Oncogene. 2009;28:2940-7.

29. Cheng GZ, Zhang WZ, Sun M, Wang Q, Coppola D, Mansour $\mathrm{M}$, et al. Twist is transcriptionally induced by activation of STAT3 and mediates STAT3 oncogenic function. J Biol Chem. 2008;283:14665-73.

30. Zang C, Liu X, Li B, He Y, Jing S, He Y, et al. IL-6/STAT3/ TWIST inhibition reverses ionizing radiation-induced EMT and radioresistance in esophageal squamous carcinoma. Oncotarget. 2017;8:11228-38. 